EPJ manuscript No.

(will be inserted by the editor)

JLAB-THY-02-43

\title{
Electromagnetic structure of the deuteron
}

\section{review of recent theoretical and experimental results}

\author{
Franz Gross \\ Jefferson Laboratory, 12000 Jefferson Avenue, Newport News, VA 23606; e-mail: gross@jlab.org
}

Received: September 13,2002

\begin{abstract}
Recent high energy measurements of elastic ed scattering support the use of a relativistic theory based on an accurate description of the $N N$ channel, but theory needed for an understanding of the high energy deuteron photodisintegration cross sections and polarization observables is not yet mature.
\end{abstract}

PACS. 25.30.B, 25.30.D, F elastic and inelastic electron scattering - 24.10.J Relativistic models (nuclear reactions)

\section{Introduction}

This talk reviews recent theoretical and experimental results for elastic electron deuteron scattering (yielding the deuteron form factors), threshold electrodisintegration ( $e+$ $d \rightarrow e^{\prime}+p+n$ where the mass of the final $n p$ pair, $W$, is only a few $\mathrm{MeV}$ above the threshold value of $\left.m_{p}+m_{n}\right)$, and high energy deuteron photodisintegration $(\gamma+d \rightarrow p+n)$. The talk is based on the complete reviews of Refs. [1, 2, 3], with a few new results not previously reported.

\section{Deuteron Wave Functions}

The deuteron wave functions are calculated from a potential (or a relativistic kernel) that has been fitted to $N N$ scattering data below lab kinetic energies of $350 \mathrm{MeV}$. Figs. 1.2, and 3 show the coordinate and momentum space wave functions for six models: Argonne AV18 [⿶, Paris [5], CD Bonn [6], IIB [7], W16 [8] and the recent Idaho potential [9]. These figures are modified versions of those in Ref. 3]; the principle change is the inclusion of the new Idaho wave functions (the heavy dashed-triple dotted line) which have a rapid but smooth cutoff above $600 \mathrm{MeV}$ momentum. This cutoff explains the ripples in the $r$ space wave functions, and will have a profound effect on the deuteron form factors.

\section{Deuteron Form Factors}

The deuteron form factors are defined by the relativistic deuteron current, which has the form

$$
\left\langle d^{\prime}\left|J^{\mu}\right| d\right\rangle=-G_{1}\left(Q^{2}\right)\left[\xi^{*} \cdot \xi\right]\left(d^{\mu}+d^{\prime \mu}\right)
$$

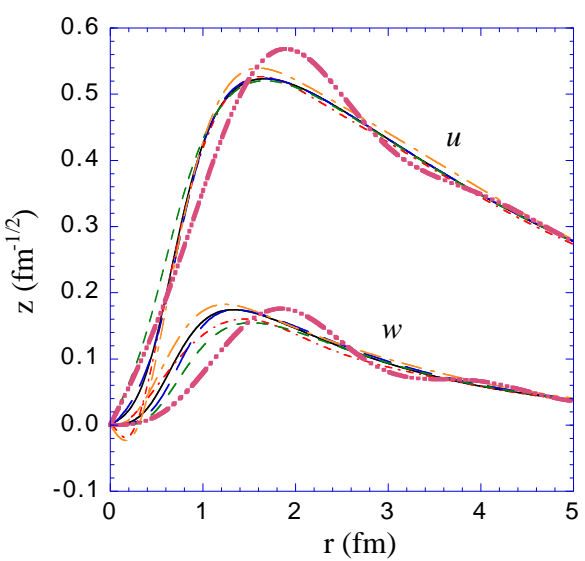

Fig. 1. The $u$ and $w$ wavefunctions in $r$ space for six models discussed in the text.

$$
\begin{aligned}
& +G_{3}\left(Q^{2}\right) \frac{\left(\xi^{* *} \cdot q\right)(\xi \cdot q)}{2 m_{d}^{2}}\left(d^{\mu}+d^{\prime \mu}\right) \\
& -G_{M}\left(Q^{2}\right)\left[\xi^{\mu}\left(\xi^{\prime *} \cdot q\right)-\xi^{* \mu}(\xi \cdot q)\right],
\end{aligned}
$$

where $Q^{2}=-q^{2}$, with $q=d^{\prime}-d$, is the square of the four-momentum transferred by the electron, and $d\left(d^{\prime}\right)$ and $\xi\left(\xi^{\prime}\right)$ are the incoming (outgoing) deuteron four momenta and polarization, respectively. Instead of $G_{1}$ and $G_{3}$, it is customary to use the linear combinations

$$
\begin{aligned}
G_{C} & =G_{1}+\frac{2}{3} \eta G_{Q} \\
G_{Q} & =G_{1}-G_{M}+(1+\eta) G_{3},
\end{aligned}
$$

with $\eta=Q^{2} / 4 m_{d}^{2}$. At $Q^{2}=0$, the form factors $G_{C}, G_{M}$, and $G_{Q}$ give the charge, magnetic and quadrupole mo- 


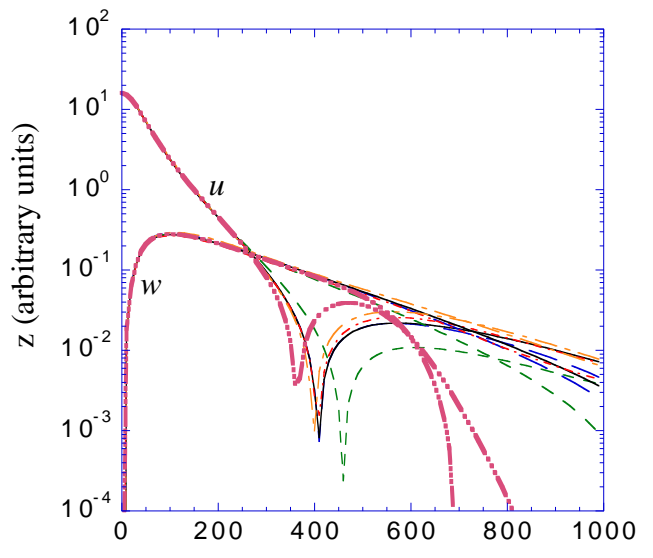

Fig. 2. The $u$ and $w$ wavefunctions in $p$ space for six models discussed in the text. (Each model is normalized so that $u(0)=$ 16.)

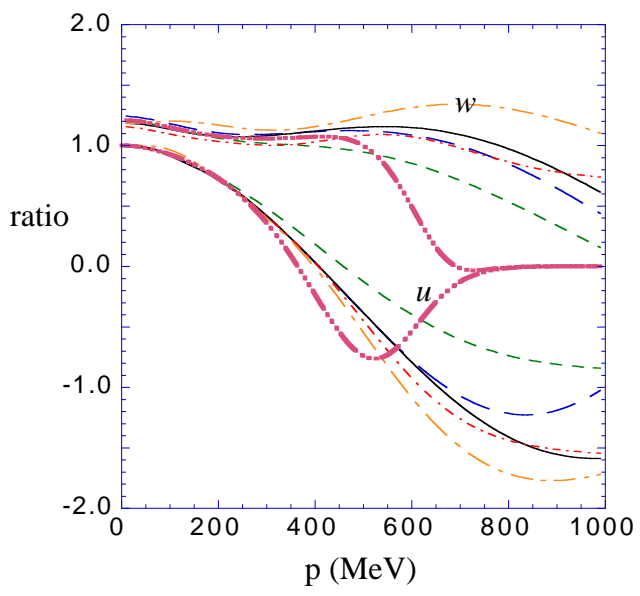

Fig. 3. The scaled $u$ and $w$ wavefunctions in $p$ space for six models discussed in the text. (The scaling function used here is defined in Ref. [3].)

ments of the deuteron

$$
\begin{aligned}
& G_{C}(0)=1 \quad(\text { in units of } e) \\
& G_{Q}(0)=Q_{d} \quad\left(\text { in units of } e / m_{d}^{2}\right) \\
& G_{M}(0)=\mu_{d} \quad\left(\text { in units of } e / 2 m_{d}\right) \text {. }
\end{aligned}
$$

The form factors must be extracted from measurements of the elastic $e+d \rightarrow e^{\prime}+d^{\prime}$ cross section, and the polarization transfer $T_{20}$, which give the combinations

$$
\begin{aligned}
& A\left(Q^{2}\right)=G_{C}^{2}\left(Q^{2}\right)+\frac{8}{9} \eta^{2} G_{Q}^{2}\left(Q^{2}\right)+\frac{2}{3} \eta G_{M}^{2}\left(Q^{2}\right) \\
& B\left(Q^{2}\right)=\frac{4}{3} \eta(1+\eta) G_{M}^{2}\left(Q^{2}\right) \\
& \tilde{T}_{20}=-\sqrt{2} \frac{y(2+y)}{1+2 y^{2}} \quad \text { with } y=2 \eta G_{Q} / 3 G_{C}
\end{aligned}
$$

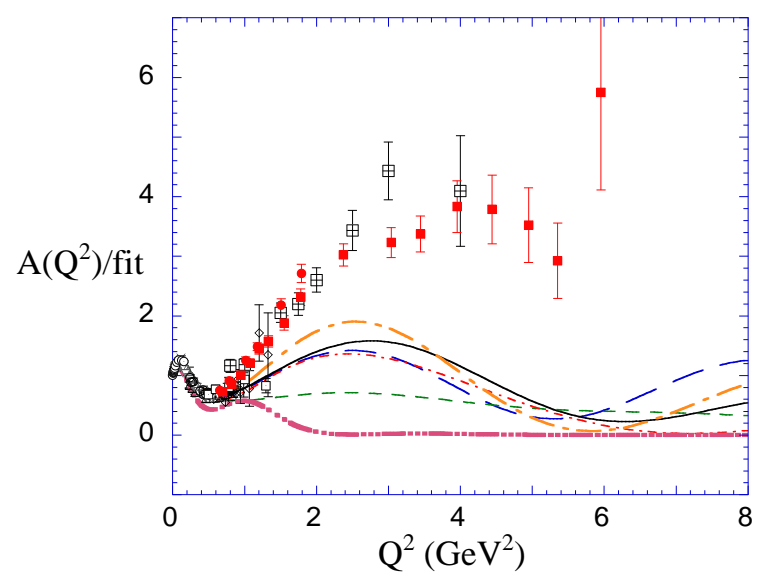

Fig. 4. The nonrelativistic inpulse approximations predictions for the six models compared to data. (The predictions and data are all divided by the scaling function given in Ref. 3].)

\subsection{Nonrelativistic Theory}

Figure 14 gives a comparison of the $A$ structure function with the theoretical predictions of the nonrelativistic impulse approximation (the NRIA includes no relativistic effects or exchange current contributions). The figure shows that the NRIA fails at large $Q^{2}$ by a factor of 4 to 8 for most of the models, and by a much larer factor for the Idaho model. Because the deuteron form factors are purely isoscalar, and isoscalar exchange currents are largely of relativistic origin, an explanation of the form factors will require a relativistic theory. The figure also shows that the Idaho model in NRIA, with no high momentum components, greatly underpredicts the $A$ structure function at high $Q^{2}$, requiring exceptionally large exchange currents in order to explain the data.

\subsection{Relativistic Theory}

The deuteron form factors are an ideal system for a test relativistic theory. The data is accurate, and the form factors are reasonably well approximated by the nonrelativistic theory of a single $N N$ channel, making them comparatively simple to calculate. Many groups have accepted the challenge and nearly all of the possible relativistic approaches outlined in Fig. 5 have been tried. The methods break into two large classes, which I call propagator dynamics (based on field theory) and hamiltonian dynamics (based on the three choices of dynamics originally classified by Dirac). The predictions of seven different relativistic calculations, listed in Table 1, are compared in Ref. 3] and in the left panel of Fig. 8 below. As shown in the table, two of these use propagator dynamics, and five use hamiltonian dynamics. Space does not permit a discussion of these approaches here; look at Ref. [3] for 
Relativity

with a fixed number of particles

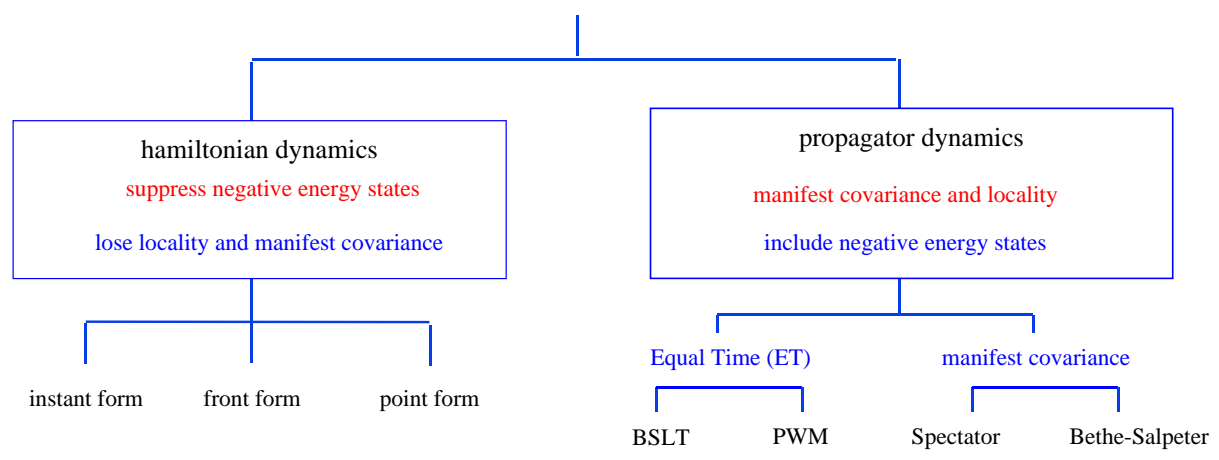

Fig. 5. Classification of relativistic methods. The class on the right is propagator dynamics $(\mathcal{P})$ and on the left is hamiltonian dynamics $(\mathcal{H})$.

a detailed discussion. Here I want to highlight some features of the VOG approach $\sqrt{10}$, which uses the manifestly covariant spectator theory [17].

In this theory the $N N$ propagator is obtained from the covariant Bethe-Salpeter propagator by replacing one of the nucleon propagators by

$$
S(p)=\frac{m+\not p}{m^{2}-p^{2}} \rightarrow 2 \pi \delta_{+}\left(m^{2}-p^{2}\right) \sum_{s} u(\mathbf{p}, s) \bar{u}(\mathbf{p}, s) \text {. }
$$

This substitution insures that the nucleon with momentum $p$ is on its positive energy mass shell, reducing the 4-d Bethe-Salpeter equation to the covariant 3-d spectator equation. The resulting relativistic wave functions depend only on the three-momentum, as in the nonrelativistic case, but can be Lorentz transformed from frame-toframe using transformations that do not depend on the details of the dynamics (i.e. they are kinematic). The boost transformation is

$$
\mathcal{B}(\Lambda) \Psi_{\alpha \lambda}^{\mu}(p, d) \xi_{\mu}=B_{\alpha \alpha^{\prime}} \Psi_{\alpha^{\prime} \lambda^{\prime}}^{\mu}(\Lambda p, \Lambda d)(\Lambda \xi)_{\mu} d_{\lambda^{\prime} \lambda}^{(1 / 2)}(\omega)
$$

where $\alpha$ is the Dirac index of the off-shell particle, $\lambda$ the helicity of the on-shell particle, and $\omega$ is the Wigner rotation angle for the helicity of the on-shell particle. This exact formula makes it possible to calculate the recoil of the outgoing deuteron exactly to all orders in $(v / c)$, and is used in calculations of the form factors. Finally, I emphasize that the close connection of this formalism to field theory makes it possible to design currents that are complete, physical, and consistent.

Figure 6 shows how experimental values of $G_{C}$ extracted from measurements of $A$ (corrected by removing the magnetic and quadrupole contributions]) compare to Sick's best fit [2], and to different relativistic calculations. Using the Argonne AV18 NRIA as a nonrelativistic standard, the data support the conclusion that relativistic effects grow rapidly for $Q \geq 0.3 \mathrm{GeV}$. Furthermore, the

\footnotetext{
1 These corrections, of the order of $5 \%$ at the larger $Q$ shown, were omitted from Ref. [3].
}

\begin{tabular}{|c|c|c|c|c|}
\hline model & class & description & in & $\begin{array}{l}\text { complete } \\
\text { current }\end{array}$ \\
\hline VOG & $\mathcal{P}$ & Spectator & yes & yes \\
\hline PWM "11 & $\mathcal{P}$ & modified & no & no \\
\hline FSR [1 & $\mathcal{H}$ & $\begin{array}{l}\text { Mandelzweig-Wallace } \\
\text { instant-form; } \\
\text { no } v / c \text { expansion }\end{array}$ & no & yes \\
\hline ARW 13. & $\mathcal{H}$ & $\begin{array}{l}\text { instant-form; } \\
\text { with } v / c \text { expansion }\end{array}$ & yes & yes \\
\hline CK $\sqrt{1}$ & $\mathcal{H}$ & $\begin{array}{l}\text { front-form; } \\
\text { dynamical light-front }\end{array}$ & no & no \\
\hline LPS 15 & $\mathcal{H}$ & $\begin{array}{l}\text { front-form; } \\
\text { fixed light-front }\end{array}$ & yes & no \\
\hline $\mathrm{AKP}$ 16 & $\mathcal{H}$ & point-form & yes & no \\
\hline
\end{tabular}

Table 1. Features of seven relativistic models.

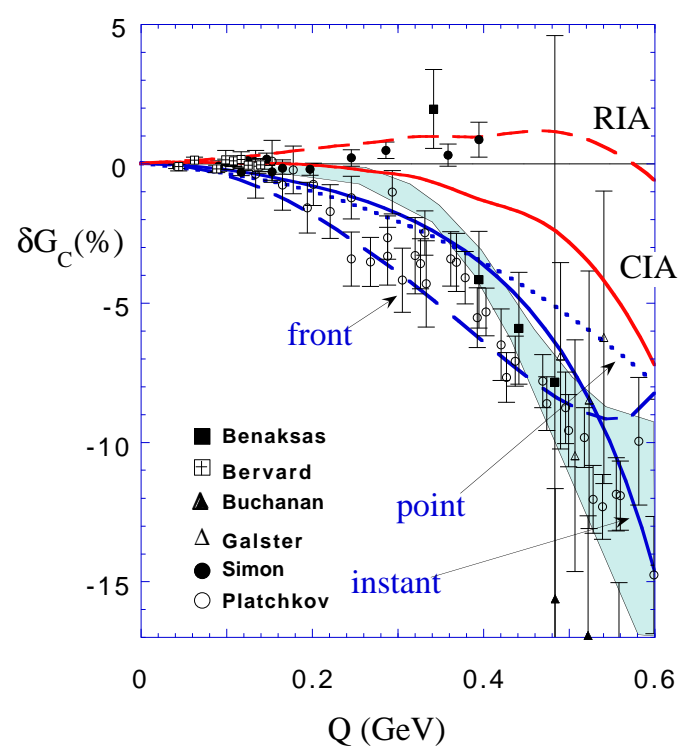

Fig. 6. Comparison of selected relativistic calculations of $G_{C}$ with charge form factor data. The shaded area is the best fit (with errors) determined by Sick [2]. All curves and data are expressed as a percentage difference from the AV18 calculation in NRIA. 

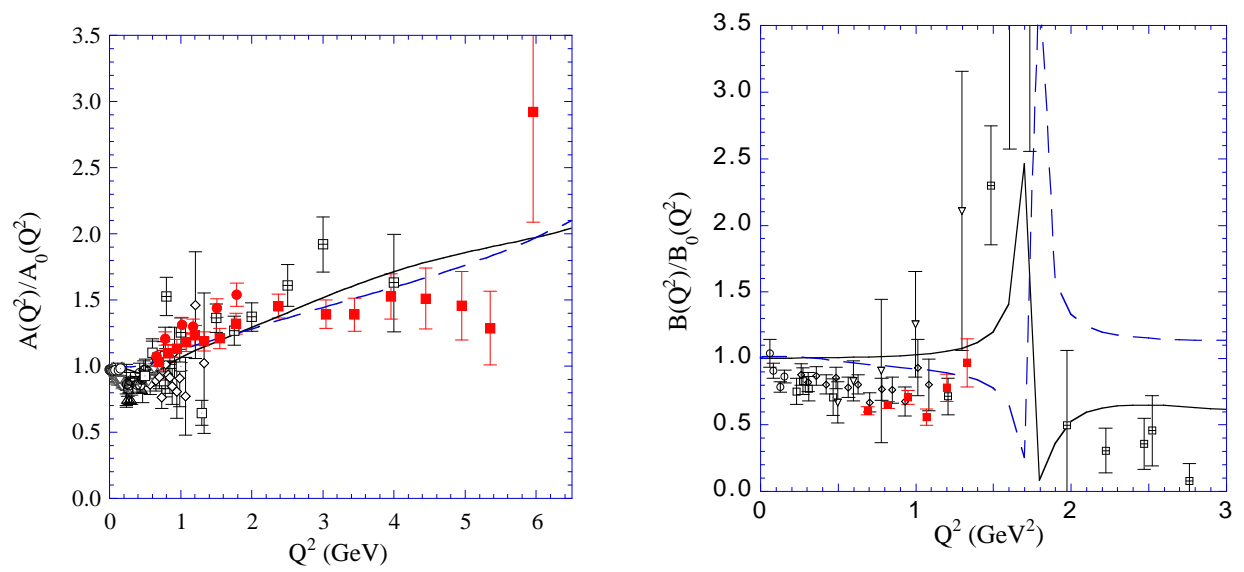

Fig. 7. Ratios of the structure functions $A$ and $B$ with different choices for the form factors $F_{3}$ or $f_{\rho \pi \gamma}$ normalized to $A_{0}$ and $B_{0}$ calculated with the "standard" choices $F_{3}=$ dipole and $f_{\rho \pi \gamma}=0$. Solid line: $F_{3}=$ tripole, $f_{\rho \pi \gamma}=0$. Dashed line: $F_{3}=$ dipole, $f_{\rho \pi \gamma}=$ dipole with $\Lambda_{d}^{2}=1.5 \mathrm{GeV}^{2}$.

accuracy of the measurements is comparable to the differences between relativistic approaches, suggesting that a precision $1 \%$ measurement of $G_{C}$ would further constrain relativistic theory 18. The apparent inconsistency between the Simon and Platchkov measurements is an additional motivation, but Sick has studied both measurements in detail and reports that the data sets are consistent within statistical and systematic errors 19 .

I will conclude this discussion by calling attention to one aspect of the VOG spectator calculation of special theoretical interest. Feynman showed years ago that a photon amplitude will be gauge invariant if (i) the coupling of the photon to the (off-shell) constituents inside the amplitude satisfies the Ward-Takahashi identities, and (ii) electromagnetic couplings to all particles inside the amplitude are included. In 1987, Riska and I found out how to generalize this result to calculations using either Bethe-Salpeter amplitudes or spectator amplitudes involving composite constituents 20]. In the VOG theory, off-shell nucleons are described by a dressed propagator $S(p)=h^{2} S_{0}(p)$, where $h=h(p)$ is a scalar function of the square of the off-shell nucleon four-momentum $p^{2}$ [with its only parameter fixed by fits to the $N N$ data], and $S_{0}$ is the undresed nucleon propagator. Use of this propagator requires that the simplest, but not unique single nucleon current must have the form

$$
\begin{aligned}
j^{\mu}\left(p^{\prime}, p\right)= & f_{0}\left(p^{\prime}, p\right)\left(F_{1}\left(Q^{2}\right) \gamma^{\mu}+\frac{F_{2}\left(Q^{2}\right)}{2 m} i \sigma^{\mu \nu} q_{\nu}\right) \\
& +g_{0}\left(p^{\prime}, p\right) F_{3}\left(Q^{2}\right) \frac{m-\not p^{\prime}}{2 m} \gamma^{\mu} \frac{m-\not p}{2 m}
\end{aligned}
$$

where $f_{0}$ and $g_{0}$ are known functionals of $h$ and $h^{\prime}=h\left(p^{\prime}\right)$ [with $p^{\prime}$ the four momentum of the outgoing nucleon], and $F_{3}(0)=1$ but is otherwise undefined. In the original calculations, $F_{3}$ was taken to be a dipole, but if it is made somewhat harder, all of the deuteron observables can be well described by the spectator theory. This remarkable result is shown in Fig. 7. Neglecting the $\rho \pi \gamma$ exchange current, and choosing $F_{3}$ to be the tripole $\left(1+Q^{2} / \Lambda_{t}^{2}\right)^{-3}$ with $\Lambda_{t}^{2}=5 \mathrm{GeV}^{2}$, brings all of the observables into excellent agreement with the high $Q^{2}$ data measured at SLAC and JLab (including $T_{20}$ not shown). Alternatively, using the "standard" dipole form, $F_{3}=\left(1+Q^{2} / \Lambda_{d}^{2}\right)^{-2}$ with $\Lambda_{d}^{2}=0.71 \mathrm{GeV}^{2}$ and choosing a dipole form for the $\rho \pi \gamma$ form factor (with $\Lambda_{d}^{2}=1.5 \mathrm{GeV}^{2}$ ) will give agreement for the structure function $A$, but increases the disagreement in $B$. I conclude that a reasonable choice of $F_{3}$ will bring the VOG model into perfect agreement with the deuteron data. Of course, it remains to see if this choice will also describe other electromagnetic processes, and this brings us nicely to the next topic.

\section{Threshold Electrodisintegration}

Deuteron electrodisintegration to an $n p$ final state with an invariant mass $W$ only a few $\mathrm{MeV}$ above the threshold, $W_{t}=m_{p}+m_{n}$, is very closely related theoretically to elastic ed scattering. Here the final scattering state can be as reliably calculated as the initial deuteron state using the same theory. If the mass of the final state is close enough to threshold, the transition will be dominated by (purely magnetic) transitions to the ${ }^{1} S_{0}$ final state (transitions to the ${ }^{3} S_{1}$ final state are suppressed by the orthogonality of the states), and the transition amplitude will be purely isovector. Together with elastic scattering (purely isoscalar) the two processes can be used to independently determine the isoscalar and isovector exchange currents.

Figure 8 compares calculations of threshold electrodisintegration with recent calculations of the $B$ structure function (the magnetic counterpart of the electrodisintegration amplitude). In spite of the close theoretical connection between these two reactions, few theoretical groups have calculated both. Both are very sensitive to the details of the theory, and together will provide a stringent test. 

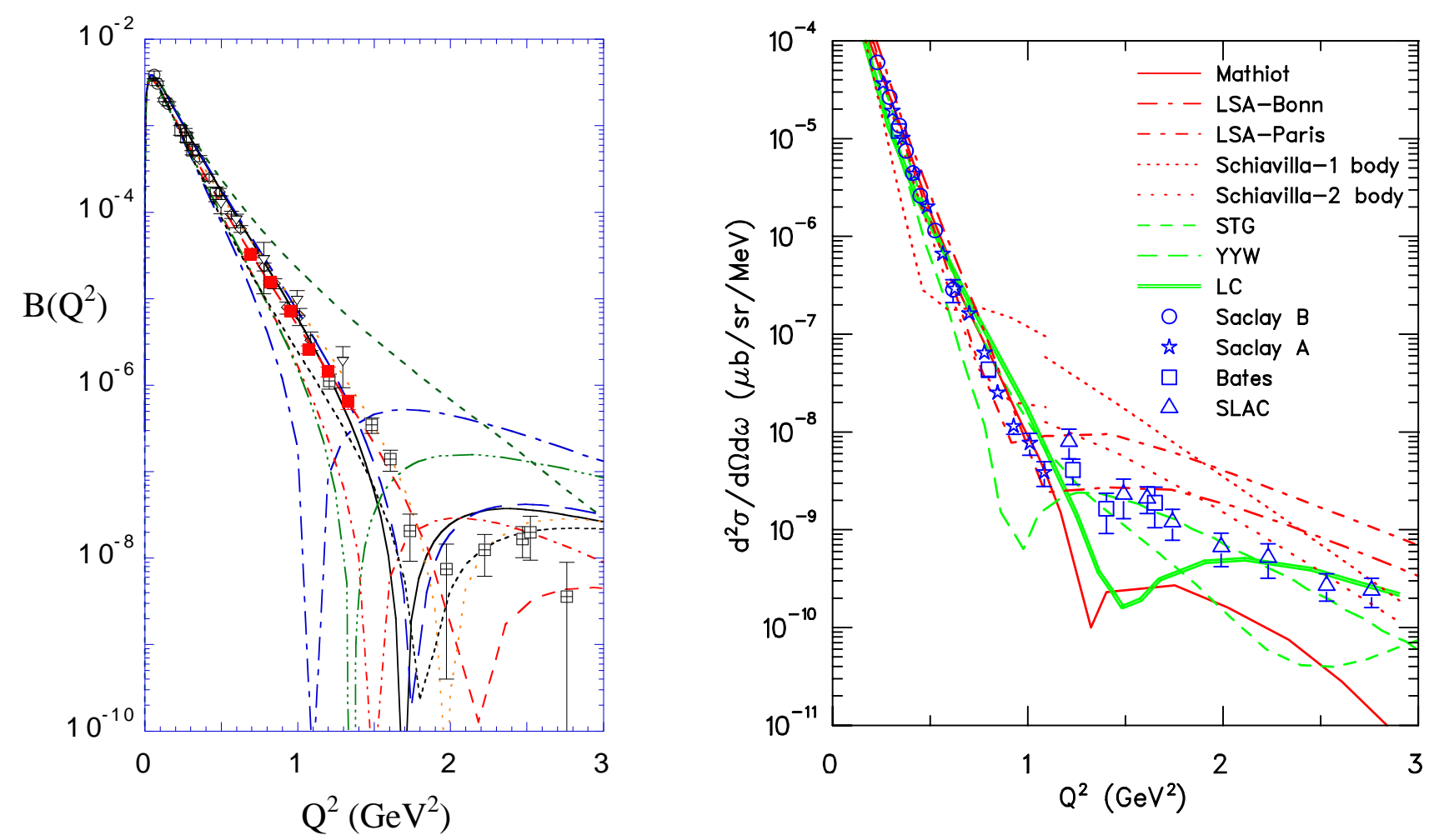

Fig. 8. Theory and data for the elastic $B$ structure function (left panel) and the threshold electrodisintegration cross section (right panel). The calculations listed in Table 1 are shown in the left panel.

More accurate data is needed for both reactions, particularly in the region of the minima near $Q^{2} \simeq 1.5 \mathrm{GeV}^{2}$.

\section{Photodisintegration}

There are significant differences between elastic ed scattering using electrons of several $\mathrm{GeV}$, discussed above, and photodisintegration by photons of several GeV. In the elastic reaction, the final state remains bound, all of the virtual photon energy going into the recoil of the final deuteron. In the VOG relativistic approach where the boost operators are purely kinematic, this recoil can be calculated exactly, and one can hope to describe the process using dynamics based on low energy physics. In photodisintegration, however, the bulk of the photon energy goes into exciting the final state, and $4 \mathrm{GeV}$ photons have sufficient energy to excite all of the nucleon resonances listed in the particle data book! A hadronic calculation of this final state is necessarily a complicated problem involving the coupling of over 200 channels. Clearly the theory of high energy photodisintegration must either be based on an average treatment of many hadronic channels, or on the use of quark degrees of freedom, which provides the high energy alternative to hadronic based descriptions.

A number of theoretical descriptions using quarks or of Reggie trajectories (a form of averaging over hadronic degrees of freedom) have been applied to the description of photodisintegration. For a discussion see the review [3]

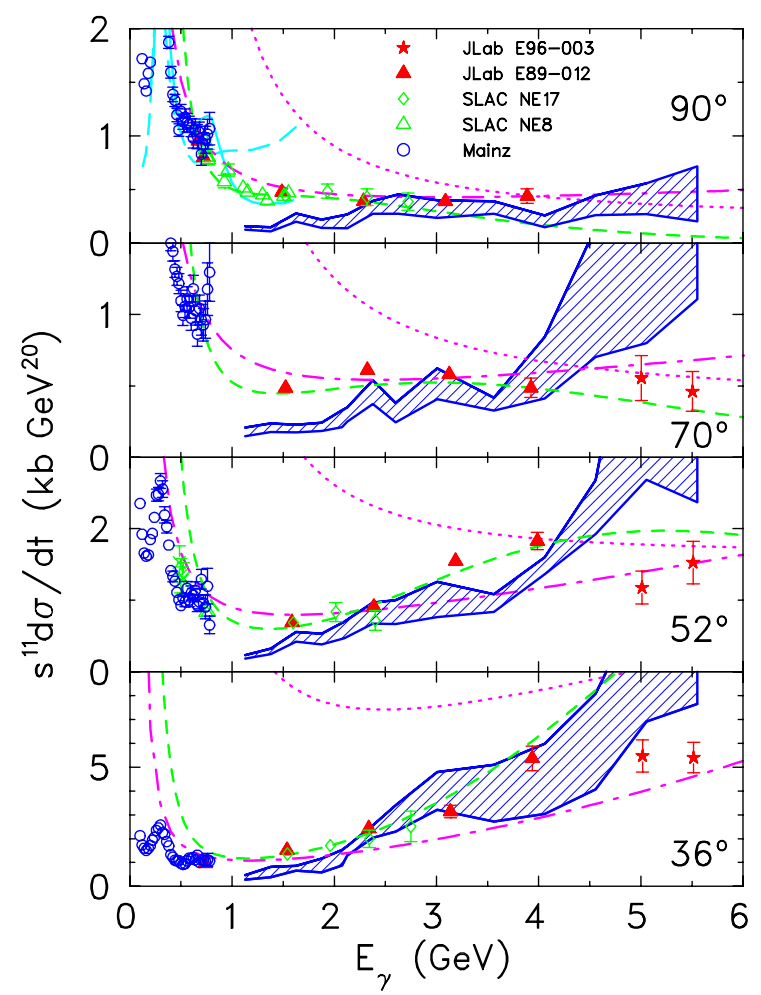

Fig. 9. Photodisintegration cross section $s^{11} d \sigma / d t$ versus incident lab photon energy for various fixed angles. For discussion and references see Ref. [3]. 


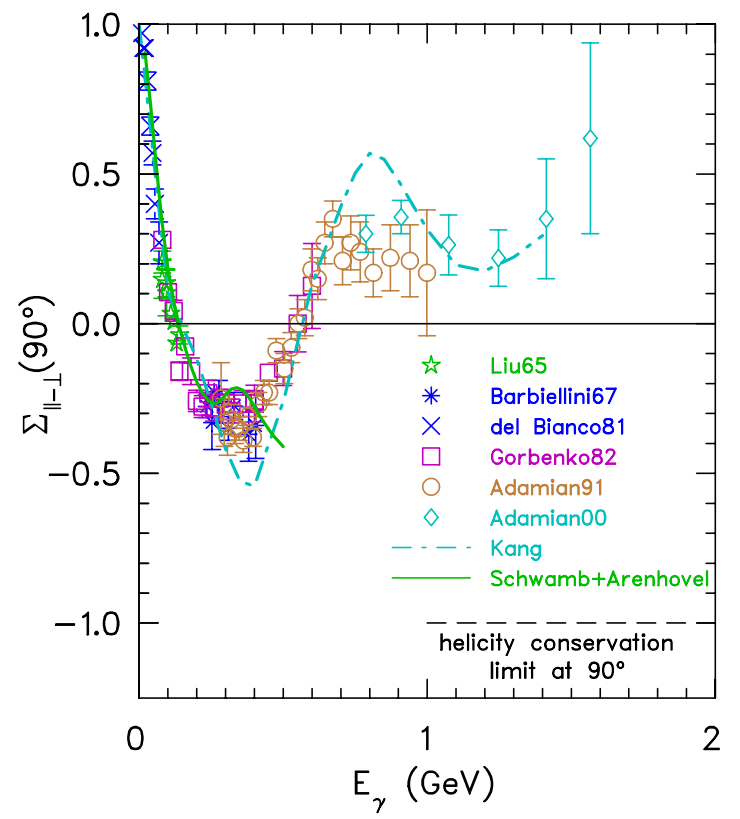

Fig. 10. Polarized photon asymmetry $\Sigma$ at $\theta_{\mathrm{cm}}=90^{\circ}$. For discussion and references see Ref. [3].

or the talk by Patrizia Rossi elsewhere in these procedings [21. Here we call attention only to the status of the prediction from perturbative QCD, that the cross section should fall like $s^{-11}$. Figure 9 shows that recent high energy data from JLab 22, 23. generally confirm this behavor, but a model by Raydushkin (the dot-dashed line) with an $s^{-10}$ behavior does just as well.

Polarization observables provide the most stringet test of any model, and pQCD in particular. Figure 10 shows recent high energy Yerevan data [24] on the polarized photon asymmetry $\Sigma$. The result expected from pQCD (supplemented by additional assumptions) that $\Sigma\left(90^{\circ}\right) \rightarrow-1$ as $E_{\gamma} \rightarrow \infty$ is not confirmed.

\section{Conclusions}

Elastic scattering and photodisintegration with $4 \mathrm{GeV}$ electrons are closely related experimentally, but require very different theoretical approaches. For example, if reasonable adjustments are made in the high $Q^{2}$ behavior of the current of an off-shell nucleon, elastic scattering is well described by a relativistic theory based on low energy $N N$ scattering. This study confirms our fundamental understanding of low energy $N N$ scattering, shows us that the relativistic theory is under control, and fixes the high $Q^{2}$ behavior of the off-shell nucleon current.

A similar understanding of high energy photodisintegration is not yet available; quark degrees of freedom, hidden in elastic scattering, may eventually be required for an understanding of photodisintegration. However, the early successes of pQCD do not seem sufficiently robust to serve as a basis for a deeper understanding in this energy range.
It is a pleasure to thank Ron Gilman for the collaborative effort leading to Ref. [3]. He supplied many of the figures in this minireview. I also thank Ingo Sick for helpful discussions and for a computer version of his form factor fits, and Ruprecht Machleidt for numerical values of his Idaho wave functions. My apologies to the many people who supplied information or whose work is not cited in this short summary; they are listed and cited in [3]. This work was supported in part by the US Department of Energy. The Southeastern Universities Research Association (SURA) operates the Thomas Jefferson National Accelerator Facility under DOE contract DE-AC0584ER40150. It also is a pleasure to acknowledge support under DOE grant No. DE-FG02-97ER41032.

\section{References}

1. M. Garcon and J. W. Van Orden, Adv. Nucl. Phys., 26 (2001) 293.

2. I. Sick, Prog. Part. Nucl. Phys., 47 (2001) 245.

3. R. Gilman and F. Gross, J. Phys. G, 28 (2002) R37.

4. R. B. Wiringa, V. G. J. Stoks, and R. Schiavilla, Phys. Rev. C 51 (1995) 38.

5. M. Lancombe, B. Loiseau, J. M. Richard, R. Vinh Mau, P. Côté, P. Pirès, and R. de Tourreil, Phys. Rev. C 21 (1980) 861.

6. R. Machleidt, F. Sammarruca, and Y. Song, Phys. Rev. C 53 (1996) R1483.

7. F. Gross, J. W. Van Orden and K. Holinde, Phys. Rev. C 45 (1992) 2094.

8. A. Stadler and F. Gross, Phys. Rev. Lett. 78 (1997) 26.

9. D. R. Entem and R. Machleidt, Phys. Lett. B 524 (2002) 93.

10. J. W. Van Orden, N. Devine, and F. Gross, Phys. Rev. Lett. 75 (1995) 4369.

11. D. R. Phillips, S. J. Wallace, and N. K. Devine Phys. Rev. C 58 (1998) 2261.

12. J. Forest and R. Schiavilla 2001 (private communication)

13. H. Arenhövel, F. Ritz, and T. Wilbois Phys. Rev. C 61 (2000) 034002.

14. J. Carbonell and V. A. Karmanov Eur. Phys. J. A 6 (1999) 9.

15. F. M. Lev, E. Pace, and G. Salmé Phys. Rev. C 62 (2000) 064004.

16. T. W. Allen, W. H. Klink, and W. N. Polyzou Phys. Rev. C 63 (2001) 034002.

17. F. Gross Phys. Rev. 186 (1969) 1448; Phys. Rev. D 10 (1974) 223; Phys. Rev. C 26 (1982) 2203.

18. See JLab approved experiment E-02-004, R. Gilman, contactperson.

19. I. Sick, private communication. I thank Ingo Sick for supplying the fit routine used in Ref. [2], and for helpful discussions.

20. F. Gross F and D. O. Riska, Phys. Rev. C 36 (1987) 1928.

21. P. Rossi, invited talk on deuteron photodisintegration, these procedings (2002).

22. C. Bochna, et.al., Phys. Rev. Lett. 81 (1998) 4576.

23. Schulte E, et.al., Phys. Rev. Lett. 87 (2001) 102302.

24. F. Adamian, et.al., Eur. Phys. J. A 8 (2000) 423. 